\title{
Resistance-based high resolution recording of predefined 2-dimensional pen trajectories in an fMRI setting
}

Citation for published version (APA):

Reithler, J., Reithler, H., Goebel, R., van Mier, H. I., \& Van den Boogert, E. (2006). Resistance-based high resolution recording of predefined 2 -dimensional pen trajectories in an fMRI setting. Journal of Neuroscience Methods, 152(1-2), 10-17. https://doi.org/10.1016/j.jneumeth.2005.08.012

Document status and date:

Published: 01/01/2006

DOI:

10.1016/j.jneumeth.2005.08.012

Document Version:

Publisher's PDF, also known as Version of record

\section{Document license:}

Taverne

Please check the document version of this publication:

- A submitted manuscript is the version of the article upon submission and before peer-review. There can be important differences between the submitted version and the official published version of record.

People interested in the research are advised to contact the author for the final version of the publication, or visit the DOI to the publisher's website.

- The final author version and the galley proof are versions of the publication after peer review.

- The final published version features the final layout of the paper including the volume, issue and page numbers.

Link to publication

\footnotetext{
General rights rights.

- You may freely distribute the URL identifying the publication in the public portal. please follow below link for the End User Agreement:

www.umlib.nl/taverne-license

Take down policy

If you believe that this document breaches copyright please contact us at:

repository@maastrichtuniversity.nl

providing details and we will investigate your claim.
}

Copyright and moral rights for the publications made accessible in the public portal are retained by the authors and/or other copyright owners and it is a condition of accessing publications that users recognise and abide by the legal requirements associated with these

- Users may download and print one copy of any publication from the public portal for the purpose of private study or research.

- You may not further distribute the material or use it for any profit-making activity or commercial gain

If the publication is distributed under the terms of Article $25 \mathrm{fa}$ of the Dutch Copyright Act, indicated by the "Taverne" license above, 


\title{
Resistance-based high resolution recording of predefined 2-dimensional pen trajectories in an fMRI setting
}

\author{
Joel Reithler ${ }^{\mathrm{a}, *}$, Hans Reithler ${ }^{\mathrm{b}}$, Erik van den Boogert ${ }^{\mathrm{c}}$, Rainer Goebel ${ }^{\mathrm{a}, \mathrm{c}}$, Hanneke van Mier ${ }^{\mathrm{a}}$ \\ ${ }^{a}$ Cognitive Neuroscience Department, Faculty of Psychology, Maastricht University, P.O. Box 616, 6200 MD Maastricht, The Netherlands \\ ${ }^{\mathrm{b}}$ Physics Institute 3A, RWTH Aachen University, Physikzentrum der RWTH Aachen, D-52074 Aachen, Germany \\ ${ }^{\mathrm{c}}$ F.C. Donders Centre for Cognitive Neuroimaging, P.O. Box 9101, 6500 HB Nijmegen, The Netherlands
}

Received 5 April 2005; received in revised form 29 July 2005; accepted 8 August 2005

\begin{abstract}
The recent advent of functional magnetic resonance imaging (fMRI) as a readily accessible neuroimaging method has led to exciting new insights into the functioning of the human motor system. However, technical complications related to the fMRI scanner environment often limit the ability to measure the desired behavioral data reflecting the subjects' movements. In order to perform kinematic registrations of predefined complex two-dimensional movement patterns while scanning, a new MR-compatible setup has been developed. The method presented here allows the recording of detailed pen tracing data during concurrent functional image acquisition. Essentially, temporally high resolved resistance measurements are used to keep track of the covered distance across time, as applied here to the tracing of various mazes. In this way, the current setup adds the close monitoring of continuous tracing movements to the spectrum of behavioral data which can be successfully obtained in an fMRI setting.
\end{abstract}

(C) 2005 Elsevier B.V. All rights reserved.

Keywords: Movement kinematics registration; MR-compatible; Pen tracing; Resistance measurement; Motor sequence learning; fMRI

\section{Introduction}

As has been commonly acknowledged, functional magnetic resonance imaging (fMRI) has become a powerful and widely used tool to study the functional organization of the brain (e.g. Menon, 2001; Savoy, 2001). When focusing on our understanding of the human motor system, its application has resulted in substantial progress in numerous areas of research (Mattay and Weinberger, 1999; Rowe and Frackowiak, 1999), despite the fact that more perception-oriented studies still dominate the neuroscience literature (Illes et al., 2003). Partially due to limitations imposed by the magnetic resonance (MR) scanner environment, research in the motor domain typically employs button-press responses as the behavioral counterpart for the imaging data being obtained. However, this strong emphasis on discrete finger movements does not do justice to the rich repertoire of actions that humans are capable of performing (Sanes, 2003).

\footnotetext{
* Corresponding author. Tel.: +31 43 3881896; fax: +31 433884125 .

E-mail address: J.Reithler@ psychology.unimaas.nl (J. Reithler).
}

Recognizing these limitations, several efforts to record additional data on movement kinematics while scanning have been reported in the literature. Examples of more sophisticated kinematic registration techniques range from angular joint motion recordings (Debaere et al., 2004) or handgrip force measurements (Liu et al., 2000), to the use of video cameras to provide visual feedback (Leube et al., 2003) and input devices like fiber optic joysticks (Miall et al., 2001) and pressuresensitive air-filled cushions (Jantzen et al., 2004). However, in order to study the learning of complex two-dimensional movement sequences as performed in more natural continuous pen movements, different requirements need to be met which go beyond the capabilities of the aforementioned techniques. Foremost, these requirements pertain to the registration of fast and detailed kinematic measurements of movements along different trajectories. Measurements along predefined 2Dpatterns were previously reported by van Mier and Petersen (2002), using a light-emitting pen and a conventional video camera placed outside the MR scanner room. To allow a more detailed tracking, the new setup introduced here was developed to improve the sampling rate and the spatial resolution in comparison to this earlier attempt, while being compact, 
having built-in calibration capabilities and being fully MRcompatible.

The need to develop a new setup was evident after confirming that the commercial graphic digitizing tablets we used in previous behavioral experiments fail to work in an MR environment. Although initially a compact and MR-compatible CCD-based optical measurement was considered, the resistancebased recording as presented here was preferred because of its much higher sampling rate at a lower cost. The presented setup enables the recording of continuous pen trajectories related to complex, mechanically predefined, two-dimensional patterns in an fMRI setting. During development, several technical challenges needed to be faced, including the handling of unavoidable pressure-related variations in the contact resistance at the tip of the tracing pen. Additionally, the device which enables the tracking of the subject's tracing movements should neither be affected by the scanner's strong static magnetic field, nor by the gradient-switching occurring during echo planar imaging (EPI) sequences. Likewise, the quality of the fMRI data should not be compromised by the operations of the new measurement device. The present paper illustrates the approach that was chosen to overcome these stringent limitations and discusses the validation data showing its feasibility in the context of a maze learning task. In this task, subjects learn to perform new movement sequences by continuously tracing along a path which is defined by a delimiting groove and which constitutes a maze consisting of contiguous straight segments. While keeping their eyes closed during tracing, subjects should solely rely on somatosensory and proprioceptive feedback to learn the novel continuous sequence (van Mier et al., 1998, 2004). By combining the recording of detailed pen tracing data and the relatively fast image acquisition of fMRI, the relation between behavioral and neural manifestations of learning can be closely monitored over the entire course of skill acquisition.

\section{Materials and methods}

\subsection{Resistance-based position measurement}

The setup's rationale takes advantage of the fact that each trajectory to be traced is basically determined in advance. By predefining the path's general two-dimensional geometry, any position along the path can consequently be represented by a one-dimensional variable which indicates the distance covered with respect to its starting point. In our setup, keeping track of the covered distance is achieved by a resistance-based measurement, where the resistance between any current pen position and the path's starting point is proportional to the distance between them.

The mazes which are used in the present application consist of successive straight-line segments which form a closed loop (Fig. 1a). The trajectory that should be followed is determined by a groove - the wide dark line in Fig. 1a - which constitutes the interconnected segments of the maze. Subjects are instructed to learn the novel movement sequence by tracing the path which is dictated by the maze's geometry as fast as possible, solely based on somatosensory information they can extract from the predefined groove and proprioceptive feedback they get while performing these new sequential movements.

The recording of the subject's pen tracing movements is essentially accomplished in the following way: at the bottom of the groove, a resistive layer forms the surface which the conductive pen tip contacts. The maze path is milled into this resistive layer, a cut is made near the origin to electrically separate the starting- and end-points, and electrodes are connected to both ends (Fig. 1b). The total resistance of the formed path has a known and constant value $\left(R_{\mathrm{t}} \approx 2.2 \mathrm{k} \Omega\right)$. As the pen, which simply constitutes a galvanic contact and is connected to a $5 \mathrm{~V}$ power supply via a current-limiting resistor $\left(R_{\mathrm{S}}\right)$, is moved along the resistive layer, the voltages measured at the electrodes placed at the starting- and end-point of the maze ( $U_{1 \mathrm{~L}}$ and $U_{2 \mathrm{~L}}$ in Fig. 1b) decrease/increase accordingly. These voltages allow the reconstruction of the resistances $R_{1}$ and $R_{2}$ between the pen's current position and the start and end of the trajectory, respectively. Noting that:

$R_{\mathrm{t}}=R_{1}+R_{2}=$ constant

the measured voltages $U_{1 \mathrm{~L}}$ and $U_{2 \mathrm{~L}}$ permit to calculate the quantity $R_{1}$ by:

$R_{1}=\frac{U_{2 \mathrm{~L}}-U_{1 \mathrm{~L}}+U_{2 \mathrm{~L}}\left(R_{\mathrm{t}} / R_{02}\right)}{\left(U_{1 \mathrm{~L}} / R_{01}\right)+\left(U_{2 \mathrm{~L}} / R_{02}\right)}$

where $R_{01}$ and $R_{02}$ are known resistances in series to the movement-dependent resistances $R_{1}$ and $R_{2}$.

Importantly, note that in Eq. (2) the contact resistance $R_{\mathrm{c}}$ does not appear. This means that by taking into account the relative contribution of both resistances $R_{1}$ and $R_{2}$, adverse effects on the reconstructed $R_{1}$ from unavoidable fluctuations in $R_{\mathrm{c}}$ between the pen and the maze's surface (e.g. varying according to the exerted pressure, or to the presence of dirt, or irregularities of the surface) are avoided or at least minimized. Conversely, the values above can also be used to reconstruct $R_{\mathrm{c}}$ :

$$
R_{\mathrm{c}}=\frac{\begin{array}{c}
U_{\mathrm{vcc}}-\left(U_{1 \mathrm{~L}} / R_{01}+U_{2 \mathrm{~L}} / R_{02}\right) R_{\mathrm{S}} \\
-\left(R_{1}+R_{01}\right)\left(U_{1 \mathrm{~L}} / R_{01}\right)
\end{array}}{\left(U_{1 \mathrm{~L}} / R_{01}\right)+\left(U_{2 \mathrm{~L}} / R_{02}\right)}
$$

and this actual measurement of the instantaneous contact resistance permits to detect detrimental contact resistance peaks ( $U_{\mathrm{vcc}}$ is the monitored supply voltage). Typical values of $R_{\mathrm{c}}$ are below $0.1 \mathrm{k} \Omega$ (see insert Fig. 2), i.e. small in comparison with $R_{\mathrm{s}}, R_{01}$, and $R_{02}$ (Fig. $1 \mathrm{~b}$ ). While loosing contact, $R_{\mathrm{c}}$ becomes anomalously large. By requiring a low value for $R_{\mathrm{c}}$, points included for further analysis are efficiently validated. In this way, the setup fulfills the necessary requirement that every contact loss is correctly recognized as such and hence does not lead to any misinterpretations. Of course, when the pen is lifted from the resistive layer, no position is measurable and the suitability of the validated data available is exclusively determined by the requirements of the data analysis.

Furthermore, the voltage recorded at the point between the pen's protective resistor and the pen's tip, $U_{\mathrm{s}}$, provides an addi- 


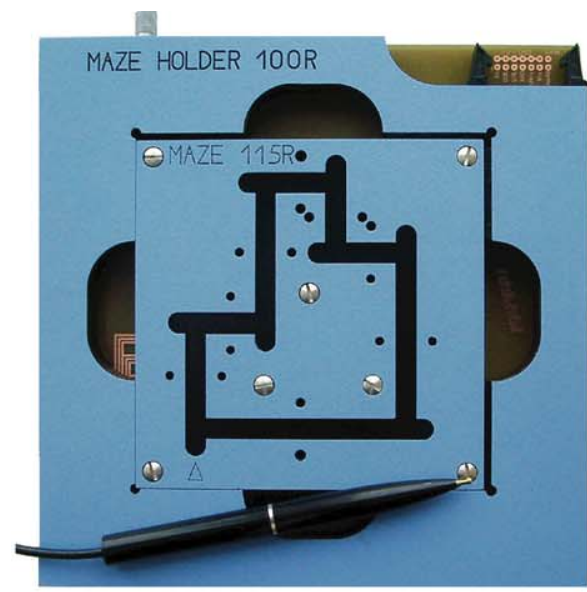

(a)

maze, maze holder and pen

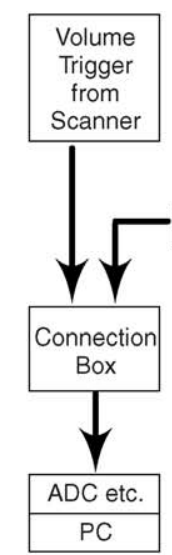

(b)

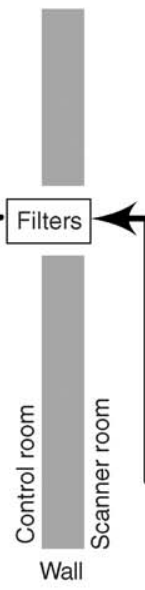

Wall

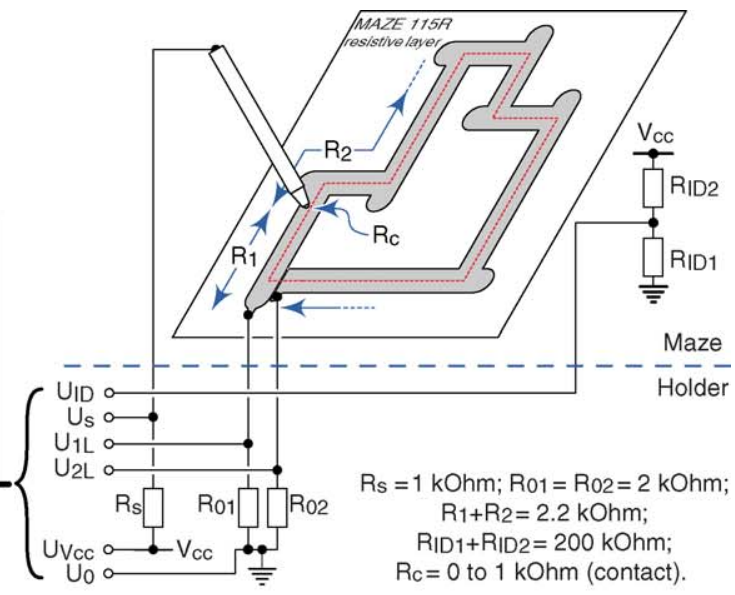

schematic setup overview

Fig. 1. (a) A maze which is plugged into the maze holder, together with the adapted pen. Several mazes of $10 \times 10 \mathrm{~cm}^{2}$ are available and readily interchangeable. The actual maze path is a $6 \mathrm{~mm}$ wide guiding groove, which is $3 \mathrm{~mm}$ deep and has a resistive layer at the bottom which is clearly visible here as a dark line. (b) A schematic overview of the setup. On the right side of the scanner room wall, the electrical maze circuit is depicted. Note that the maze path acts as a resistor $\left(R_{1}+R_{2}\right)$, and that the variable contact resistance $\left(R_{\mathrm{c}}\right)$ is in series to the protective resistor $R_{\mathrm{S}}$. The components below the horizontal dashed line are located inside the maze holder, including some filtering capacitors not shown here. The connection box and the A/D converter can be found outside of the scanner room (on the left; see text for details). Used nomenclature for measured signals: $U_{\mathrm{vcc}}$, monitoring of supply voltage $V_{\mathrm{cc}} ; U_{1 \mathrm{~L}}$, voltage measured at starting point; $U_{2 \mathrm{~L}}$, voltage measured at end point; $U_{\mathrm{s}}$, voltage measured at pen, as additional constraint; $U_{\mathrm{ID}}$, voltage for online maze identification.

tional constraint, as visible from the relation

$\frac{U_{\mathrm{vcc}}-U_{\mathrm{s}}}{R_{\mathrm{S}}}=\frac{U_{1 \mathrm{~L}}}{R_{01}}+\frac{U_{2 \mathrm{~L}}}{R_{02}}$

which yields a continuous monitoring of the measurement accuracy. At a current of about $1.7 \mathrm{~mA}$, the two values measured agree to $(1 \pm 0.22) \%$.

Based on a calibration procedure carried out previously (see Section 3), the reconstructed resistances are finally transformed into a measure of the covered distance by:

$L_{1}=\left(\frac{L_{\mathrm{t}}}{R_{\mathrm{t}}}\right) R_{1}+L_{0}$

where $L_{0}$ is a constant offset whose effective value is related to the shape of the ends of the resistive strip. An example of the resulting $L_{1}$ curves that can be obtained is shown in Fig. 2.

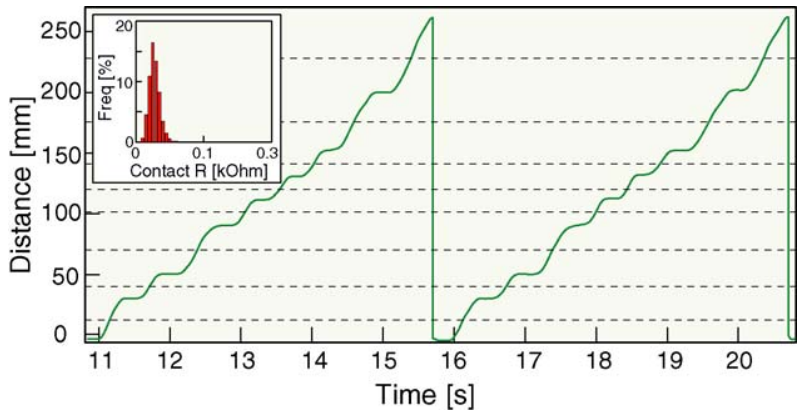

Fig. 2. The reconstructed pen position is shown as a function of time for two consecutive loops through the maze (preprocessed data from a run during EPI image acquisition). The dashed lines indicate the location of the eight calibration positions, which also constitute the boundaries of the functional segments during data analysis. Furthermore, note the short plateaus which are related to the slowing down in the corner regions. The insert on the left shows the distribution of the contact resistance values for a run in which six loops were performed.
The basic components of the system needed to realize the resistance-based recording, and further information about the acquisition process itself are discussed in detail in the next sections.

\subsection{Setup components}

\subsubsection{Mazes}

A series of five mazes was designed. Irrespective of their individual geometries, all mazes are composed of eight straight segments that form a $260 \mathrm{~mm}$ long path. An example is shown in Fig. 1. At each intersection, a so-called 'dead-end' is included to enforce a choice with regard to the direction in which to proceed. Moreover, the end of the maze path coincides with its starting point, constituting a closed loop which can be traced continuously (van Mier et al., 1993).

Each maze consists of four separate layers: The plastic maze layer on top contains a $6 \mathrm{~mm}$ wide, precision-milled groove that defines the trajectory to be traced. Furthermore, a pair of holes is present half way on each segment, to position a small tool permitting calibration of the data by measuring at predefined reference positions. The second layer consists of a $0.12 \mathrm{~mm}$ thin, resistive carbon-loaded plastic foil (Makrofol KL3-1009, Bayer, Leverkusen, Germany), machined along the outline of the maze path. Prior to machining, the resistive foil has been glued onto the third layer, which is a rigid printed circuit board (PCB) that also holds the pins which establish the contacts of both ends of the resistive foil to the maze holder (see next section). Furthermore, the PCB holds two resistors which serve to identify the maze online, as well as their respective contact pins and a contact pin to the shielding ground of the PCB. Finally, a thick plastic base plate at the bottom houses all contact pins and has four 'key' holes which help to unambiguously orient the maze when inserting it into the maze holder, without endangering any of its contacts. 


\subsubsection{Maze holder}

The mazes can be plugged into the maze holder (Fig. 1a), where the remaining leads, resistors, capacitors and connectors that complete the setup's intrinsic wiring are located. The maze holder also consists of four assembled layers, of which the PCB layer holds the female contacts for the maze's signal pins, together with further protective resistors and filtering capacitors. Additionally, it includes connectors for the pen and for the readout and voltage supply cable. By design, the maze holder offers the flexibility which is necessary to efficiently switch between mazes within a few seconds.

\subsubsection{Pen}

The tracing is performed with a conductive pen, which is an integral part of the setup's electrical circuit (Fig. 1). It was adapted to provide the necessary connection to the maze holder, without sacrificing the natural feel of a pen. The pen's tip is a $2 \mathrm{~mm}$ diameter conductive sphere, which can be smoothly moved along the maze path.

\subsubsection{Cabling, $R F$ filter and connection box}

A $9 \mathrm{~m}$ (inside scanner room) plus $3 \mathrm{~m}$ (outside scanner room) long shielded twisted-pair cable is used for readout of the measured signals and to provide supply voltage to the maze. The cable shield, including its extension underneath the maze, is connected to the shielding wall of the scanner room to shield the maze signals from the MR-related ones. To further avoid the leakage of high-frequency noise into and out of the scanner room, each line separately passes a custom-built RF low-pass filter which has been mounted on the MR system's Faraday cage (pass band: DC-30 kHz).

A purpose-built connection box finally serves to route the signals from the maze, the MR scanner and the interface board output to the multi-channel A/D converter section of the interface board (Fig. 1b).

\subsubsection{A/D converter and computer}

Data acquisition is performed with a multi-channel, 16bit A/D converter sampling at $200 \mathrm{kHz}$ (model NI PCI-6014; National Instruments, Austin, USA). A range of $0-5 \mathrm{~V}$ is covered across all channels, resulting in a resolution of $0.1 \mathrm{mV}$. The A/D converter interface board is installed in a PC (Optiplex GX260, Dell), with $512 \mathrm{MB}$ RAM and a $2.4 \mathrm{GHz}$ processor, running data acquisition and display software developed with LabVIEW v7.0 (National Instruments, Austin, USA) under Windows XP.

\subsection{Data acquisition and processing}

Simultaneous to the measurement of $U_{1 \mathrm{~L}}$ and $U_{2 \mathrm{~L}}$ (at the respective ends of the maze path) and $U_{\mathrm{s}}$ (at the point between the pen's protective resistor and the pen's tip), the $5 \mathrm{~V}$ input to the pen $\left(U_{\mathrm{vcc}}\right)$ and the voltage of the identification resistors of the currently traced maze $\left(U_{\mathrm{ID}}\right)$ are monitored.

For offline synchronization between tracing data and MR images, the scanner's pulses which mark the start of each multislice MR volume acquisition are fed into the sixth analog channel of the A/D converter. Since phases of tracing activity and rest are alternated during the fMRI measurements, a high or a low tone which is issued via the computer's audio output tells the subject when to start or stop tracing, respectively. In order to time-lock the alternation between these phases to the MR volume acquisition, the scanner's volume triggers are fed into a counter on the interface board which switches its output state after a predefined number of pulses. This output state transition is used to generate the instructional tone for the subject, and the counter's output level is passively added to the input of the sixth analog channel mentioned earlier for offline synchronization.

For all six analog channels mentioned above, acquisition takes place in differential mode, implementing an averaging procedure across 10 successive samples (out of sets of 50) per logged data point. The 10 samples used for 1 data point correspond to a measuring time of $0.3 \mathrm{~ms}$ and are acquired every $1.5 \mathrm{~ms}$. Typically, the obtained standard deviation across the averaged 10 samples is well under $1 \mathrm{mV}$, although in a small fraction of disturbed data points much larger values can be observed. Together with the averages, the standard deviations are also logged, as they are indicative of the data quality at this local level. A cross-check at the filtering phase has confirmed that data points discarded during this stage also initially had a large standard deviation in the raw samples. At an effective event logging rate of $\sim 666 \mathrm{~Hz}$ per channel, the raw data are written to an ASCII file for subsequent processing.

Data preprocessing involves the following steps: exclusion of samples with too large $(\geq 4 \mathrm{k} \Omega$ ) contact resistance values (constituting a small fraction of the entire distribution, see insert Fig. 2), exclusion of samples where $U_{1 \mathrm{~L}}+U_{2 \mathrm{~L}}<2 \mathrm{~V}$ indicating (partial) contact losses, noise peak detection by determination of signal smoothness, and FFT-based low-pass filtering. Whenever a sample is discarded during preprocessing, linear interpolation is used to replace the affected data point, based on the fact that the high sampling rate ensures that neighboring samples are clustered closely enough in space to render linear interpolation a valid method. Furthermore, the reconstruction of the pen's position $\left(L_{1}\right)$ across time is obtained from the $R_{1}$ values. The value of $R_{1}$ (Eq. (2)) is reconstructed from the values of $U_{1 \mathrm{~L}}$ and $U_{2 \mathrm{~L}}$, measured at each time point, and the constant values $R_{01}, R_{02}$, and $R_{\mathrm{t}}$. Similarly, the setup's redundant measurement feature permits to obtain two further nearly independent reconstructions of $R_{1}$, based on the values of $U_{\mathrm{s}}$ and $U_{\mathrm{vcc}}$, together with either $U_{1 \mathrm{~L}}$ or $U_{2 \mathrm{~L}}$. The values of the position obtained by these additional reconstructions agree with the value from Eq. (2) to better than $0.31 \pm 0.16 \mathrm{~mm}$ at every point, which is indicative for the overall measurement accuracy. At points disturbed by a partial contact loss, the difference can increase to up to $1.5 \mathrm{~mm}$ because the two further reconstructions are more affected than the differential method based on Eq. (2), which is used per default. To complete the preprocessing procedure, these reconstructions are thus compared with the main reconstruction to determine the overall data consistency and measurement accuracy; an average difference below $0.6 \mathrm{~mm}$ is taken as a criterion for goodness.

During subsequent data processing, relevant behavioral parameters are extracted separately for each traced segment. The main performance variables extracted from the measured local velocities are the mean velocity achieved per segment, the num- 
ber of identified stops, and the level of isochrony across different segment lengths. One option for the segment-wise report of these variables is based on a segmentation scheme in which the border of a functional segment is located in the middle of the maze's straight-line segments. This location is advantageous compared to the more natural straight-line segments' ends because the exact time of passage through a corner boundary is less well defined, both due to the subject's possible errant movements in this region and to a larger uncertainty in the position reconstruction. Conversely, the extraction of relevant parameters can also be performed for the straight-line segments by defining their boundaries to lie near the known corner regions, and hence distinguishing the achieved velocity along a straight segment from the decision time at each corner. A further calibration tool (similar to the one shown in Fig. 3) serves to obtain an accurate measurement of the necessary reference points at these boundary locations. In addition to the change in direction and the deadend at each corner - which already impede simply sliding along the borders without learning the maze shape - the use of the borders as a fast guidance along the straight lines can further be penalized by having wave-shaped borders. To perform both the preprocessing and the extraction of relevant behavioral parameters on a segment-by-segment basis, we developed a program and a graphical user interface with Matlab (v6.5 R13; The Mathworks, Natick, USA).

\subsection{Validation process}

The validation of the described setup took place in three successive steps. Initially, the question whether the reconstructed resistances could accurately reflect the covered distance along the maze path was addressed in a series of calibration measurements. Subsequently, the impact of the potential mutual interference between the maze setup and the MR scanner needed to be assessed. Therefore, the calibrations were repeated in an MR environment, together with the recording of actual loop tracing data. Data collected outside of the scanner room were used as a baseline reference to which the other measurements could be compared. Finally, the quality of the functional MR images needed to be ensured by showing that the maze setup would not interfere with the scanner's functioning. On-site tests of the setup in an MR environment were mainly performed on a $1.5 \mathrm{~T}$ whole-body system and later extended to a $3 \mathrm{~T}$ scanner setting (Magnetom Sonata and Trio, Siemens Medical Systems, Erlangen, Germany). Whenever functional images were acquired, a standard EPI sequence was used (typical settings: $\mathrm{TR}=2000 \mathrm{~ms}$; TE $=40 \mathrm{~ms} ; 90^{\circ}$ flip angle; 25 slices with $3.5 \mathrm{~mm} \times 3.5 \mathrm{~mm} \times 4.5 \mathrm{~mm}$ voxel size and a $224 \mathrm{~mm} \times 224 \mathrm{~mm}$ field of view (FoV)).

\section{Results}

\subsection{Calibration measurements}

As first calibration runs outside of the scanner, data were recorded from eight predefined positions along the maze path (Fig. 3a), making use of a tool which was designed to accurately trace these positions perpendicularly to the general tracing direction (Fig. 3b). On the one hand, the resulting data are a direct measure of the spatial resolution achieved. On the other hand, the linear relation between the measured resistance and the covered distance can be shown and quantified.

While holding the pen at a given fixed position without moving it, the observed fluctuations for the measured resistance were within a range of $\sim 0.0005 \mathrm{k} \Omega$ (i.e. about $0.02 \%$ of the total maze path resistance of $2.2 \mathrm{k} \Omega$ ). When tracing movements from the inner to the outer maze border and vice versa were performed at a given distance from the origin, the variations increased to a range of up to $\sim 0.0015 \mathrm{k} \Omega$, i.e. $\sim 0.07 \%$ of the total resistance and about $0.2 \mathrm{~mm}$ in reconstructed position, reflecting the uncertainty due to the maze path's width (insert Fig. 3a). These geometrically induced variations are treated as fluctuations in the real tracing measurements, given that no information is available on where the pen was actually passing with respect to the maze path's borders. The range of $0.0015 \mathrm{k} \Omega$ is then treated as an error of $\pm 0.00075 \mathrm{k} \Omega$, i.e. as a standard deviation of $0.1 \mathrm{~mm}$. Despite these increased fluctuations related to the maze geometry, the observed findings still clearly point to a sub-millimeter resolution for the ultimately calculated distances.

The reproducibility was obtained by repeating the calibration measurements, where the measured resistances were found to agree within $0.0005 \mathrm{k} \Omega$ on average. The absolute precision at the calibration points is obtained by folding the reproducibility with the error from fluctuations, leading to $0.0009 \mathrm{k} \Omega$ or $0.11 \mathrm{~mm}$.

Another important result is that the calibration data show a strong linear interrelation between the measured resistance and the covered distance with respect to the maze's starting point. Imposing an exact linear relation between the two quantities leads to residuals of about $1.2 \mathrm{~mm}$ rms. In other words, the pen's location can be reliably extracted from the measured signals along the entire path. Besides, the calibration procedure would assure the correctness of the performed measurements even in the light of slight local deviations from this generally applying strict linear interrelation, because the reference points for calibration are located at the points which are mainly used for extraction of the performance parameters during later runs.

\subsection{Effect of MR environment on tracing data}

To verify the setup's proper functioning in an MR environment, the calibration runs were repeated inside the scanner room. The measurements were performed at the scanner-bore's opening, about an arm-length away from the magnet's iso-center. When compared to the measurements outside of the scanner room, the observed fluctuations for the eight predefined calibration positions along the maze were unchanged in the static magnetic field of the scanner, but were $\sim 1.5$ times larger whenever EPI images were acquired. The precision at the calibration points is therefore worsened to about $0.2 \mathrm{~mm}$. Nevertheless, in all cases, the pen tip location could be determined with at least millimeter resolution (corresponding to about $0.008 \mathrm{k} \Omega$ ) after preprocessing. In terms of spatial resolution, the setup therefore clearly meets the necessary requirements for the extraction of detailed behavioral data. In addition, the earlier identified strong 

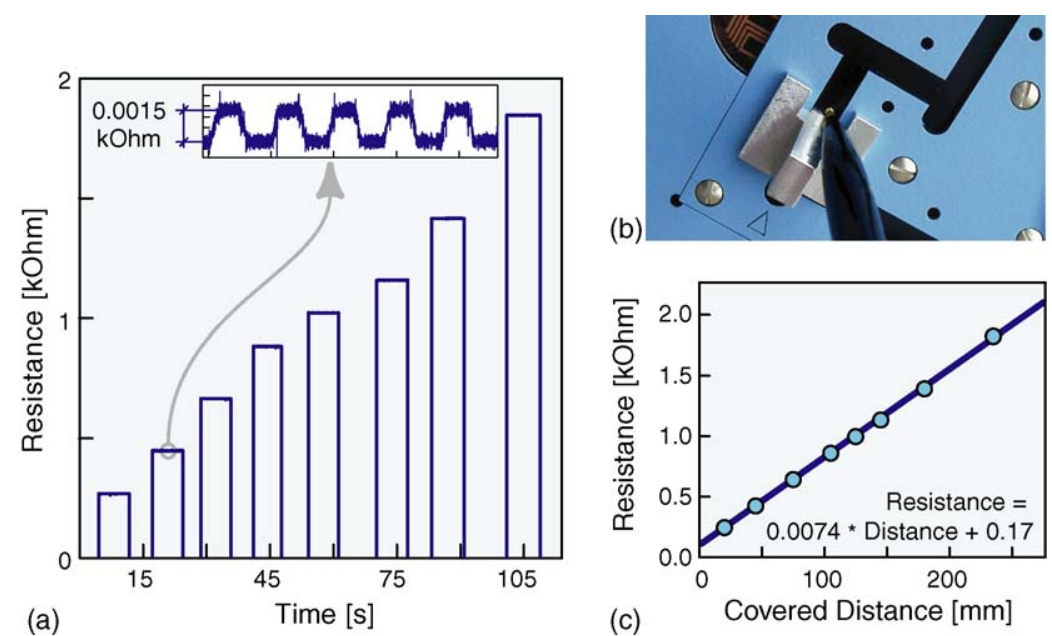

Fig. 3. Calibration data. (a) Resistances for eight predefined positions recorded outside of the MR scanner room. The insert indicates that the variations observed are mostly due to the maze path's width (showing five repetitions of going back and forth between the maze's inner and outer border at the second calibration position). Note that the reconstructed value of the maze resistance can fluctuate largely and even drop below zero whenever the pen is lifted; such points are characterized by large $R_{\mathrm{c}}$ and low $U_{1 \mathrm{~L}}+U_{2 \mathrm{~L}}$ values and are safely removed by the filtering procedure. (b) A photograph illustrating the use of the calibration tool. The small holes visible half way on each segment ensure precise positioning of the calibration tool, which serves to move the pen across the path's width at predefined positions. (c) The linear relation between covered distance and measured resistance for the eight calibration positions, as obtained from measurements during EPI image acquisition.

interrelation between reconstructed resistance on the one hand, and predefined distance on the other, remained unchanged in the MR scanner's proximity (Fig. 3c). Similar to the results obtained outside of the scanner room, residuals of about $1.2 \mathrm{~mm}$ rms were observed in both the $1.5 \mathrm{~T}$ and $3 \mathrm{~T}$ scanner settings.

When considering actual tracing data, where continuous loops are repeatedly traced through a given maze, the first observation is that one can unambiguously identify the resulting pattern as the traced trajectory (as already illustrated in Fig. 2). Yet, two recurring types of perturbation are recognizable in the raw data. First, the constantly superimposed noise that is related to measurements during EPI image acquisition is moderate, but clearly visible (Fig. 4a). A far more drastic source of noise are sporadically occurring partial or full contact losses between the pen and the traced surface, which can arise in either setting (i.e. both inside and outside of the scanner room). Either caused by a confined roughness of the maze's resistive layer or merely by the subject's movements, these con- tact losses can lead to considerable noise peaks. However, due to the redundant measurement permitting to cope with a varying contact resistance, and the high data acquisition rate at which the maze signals are recorded, the preprocessing procedure can successfully remove these undesirable fluctuations and still yield a detailed tracking even in extreme cases (Fig. 4b). In general, no more than $10 \%$ of the samples acquired during tracing are discarded.

\subsection{Effects of tracing setup on MR image quality}

The results discussed so far have dealt with the validation of the method per se, and the proper functioning of the resistive setup in the MR scanner's proximity. Conversely, there is also a need to establish that the introduced setup does not adversely impact the scanner's functioning. To assess potential effects on functional image quality, several test measurements were performed.
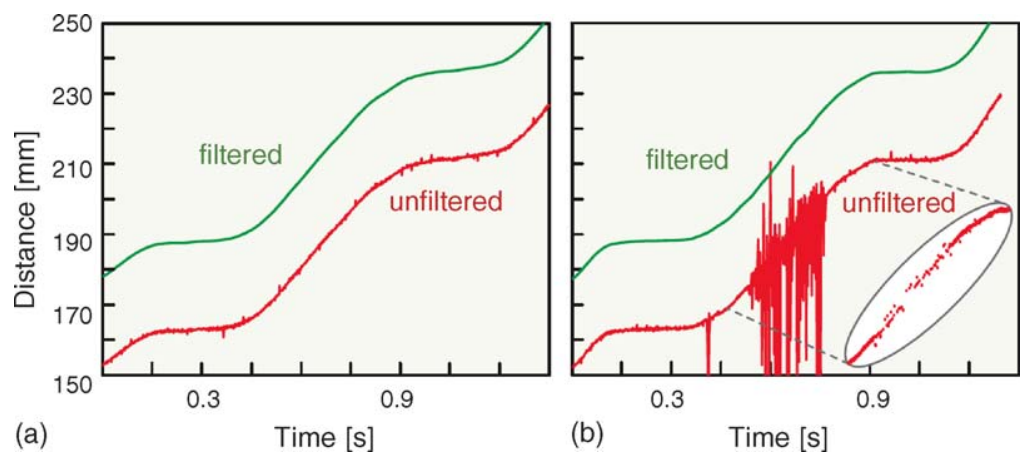

Fig. 4. Enlarged part of a measurement, illustrating generally observed noise levels. (a) The typical noise visible on raw data (red curve) is modest and is filtered out effectively (green curve, displaced vertically). (b) Even extreme disturbances of raw data points (red curve), related to loosing contact, can be filtered out without significant distortion (green curve, displaced vertically). The insert on the right shows the raw values of the points passing the filtering procedure. Due to the redundant measurement and the high sampling rate, sufficient undisturbed data points $(\sim 33 \%)$ are still available for reconstruction in this affected region. 
Using a phantom, the signal-to-noise ratio (SNR) was dynamically determined according to the procedure described by Weisskoff (1996). The first result concerned the 'best' SNR, which is defined as the ratio between the average of the mean signal intensity within a relatively large region of interest (ROI) in the middle of the image on the one hand, and the corrected average of the standard deviation of the intensity within a background noise ROI on the other hand, across all acquired functional volumes. With respect to this 'best' SNR, the measurements in the baseline condition $\left(\mathrm{SNR}_{0}=146\right)$ and the condition in which resistive measurements were performed with and without tracing $\left(\mathrm{SNR}_{0}=148\right)$ did not differ significantly in the light of generally observed $\mathrm{SNR}_{0}$ fluctuations (a mean $\mathrm{SNR}_{0}$ of 144 (S.D. $=2.9$ ) was found across 35 quality assurance measurements ranging from four months prior to three months after the measurement discussed here). Furthermore, calculating the relative fluctuations across scans for several ROIs with increasing linear size (Fig. 5) for both conditions also indicated that the presence and use of the maze setup components did not affect the scanner's stability with regard to functional measurements. The fluctuations in the baseline run amounted to $0.61,0.12$ and $0.09 \%$ of the mean signal intensity for the $1 \times 1,7 \times 7$ and $15 \times 15$ pixel ROIs, respectively (Fig. 5a). While the maze setup was present and in use, the observed fluctuations added up to $0.59,0.15$ and $0.11 \%$ for the same ROIs (Fig. 5b).

Additionally, a 'spike check' procedure which is part of the scanner's quality assurance protocols was executed. No spikes which might point to additional RF-interference patterns related to the operation of the tracing setup could be identified.
Finally, functional images were acquired during an actual experimental run involving a single subject. The run consisted of two alternating conditions, presented in a blocked fashion: an active tracing condition and a baseline rest condition, each lasting $30 \mathrm{~s}$. A switch between conditions was indicated by one of two tones generated with the LabVIEW software. In total, six repetitions of active tracing were performed. The maze setup was installed as described in the previous sections. The maze holder was fixed on a custom-built MR-compatible table. Before starting the experiment, the position of the table was adjusted so that the subject could perform the required movements rather effortlessly. Tracing was performed with the dominant right hand. The obtained imaging data were preprocessed and analyzed using BrainVoyager QX (Brain Innovation, Maastricht, The Netherlands). The resulting motion correction parameters indicated that head motion was restricted to less than $1.5 \mathrm{~mm} / 1.5^{\circ}$ in any of the possible translation/rotation directions. The deviations induced by phasic task-correlated motion were minimal $\left(<0.5 \mathrm{~mm} / 0.5^{\circ}\right)$ and do not confound any learning-related changes in brain activity which might be observed over time. When contrasting the active tracing with the baseline rest condition, a network of activated regions including the primary motor cortex (M1), supplementary motor area (SMA), pre-motor area (PMA), posterior parietal areas and the cerebellum was identified (Fig. 5c), in accordance with general expectations based on earlier work (van Mier et al., 1998). Close inspection of the time courses from selected ROIs furthermore indicated that the simultaneous recording of the subject's tracing movements did not have any noticeable effect on the quality of the functional images. This

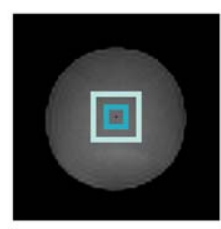

(a)

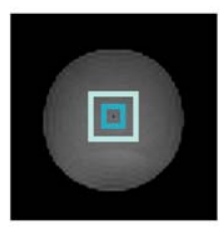

(b)

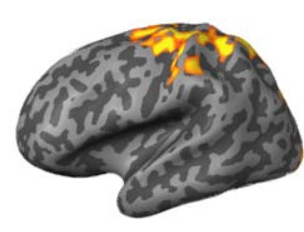

(c)
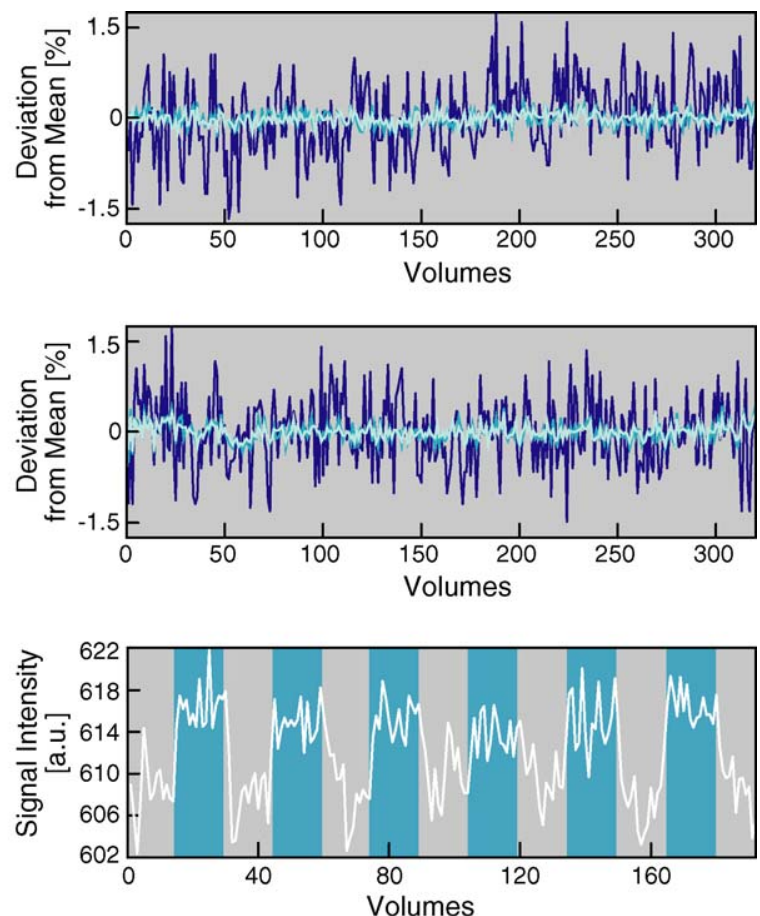

Fig. 5. Phantom slice from the stability measurements (a) without and (b) with the maze setup present and in use. The corresponding plots on the right show the deviation from the mean signal intensity for three different ROIs: a $1 \times 1$, a $7 \times 7$ and a $15 \times 15$ pixel ROI (depicted in dark to light blue, respectively), indicating that the deviations are similar in range in the two conditions, and that fluctuations decrease with increasing ROI size as expected. The lower panel (c) shows the left hemispheric activation during tracing, together with the extracted time course for M1 (light grey indicates rest condition). Note that no adverse effects due to the tracing setup's operation are observable in the fMRI time series. 
observation was further substantiated by successful application of the resistive tracing setup in the $3 \mathrm{~T}$ MR scanner environment.

\section{Discussion}

Generally, the interpretation of neuroimaging data heavily relies on concurrently recorded behavioral measurements. Unfortunately, the MR scanner environment poses severe constraints on the approaches that can be used to collect behavioral data while scanning. Whereas simple discrete button-press responses are recorded in the majority of studies on motor control and learning, several complementary kinematic registration techniques have been recently reported in the literature. In order to further enlarge the diversity of available kinematic measurements, the resistive setup presented here was developed to enable the recording of continuous pen tracing data along predefined trajectories in an fMRI setting. In the present setup, reconstruction of the pen's position across time is performed with high spatial resolution of $0.2 \mathrm{~mm}$ and temporal resolution of $0.3 \mathrm{~ms}$, is independent from the varying contact resistance between the pen and the resistive surface, and is further ensured by a redundant measurement scheme and built-in calibration features. To the best of our knowledge, no alternative setup suitable for such fast and precise measurements has been reported. Furthermore, the setup can be designated 'MR-compatible' in the sense that it is MR safe, it operates as designed in the MR environment, and finally does not have any negative effects on the image quality of the acquired fMRI scans. Although the present work focuses on the applicability to the measurement of more complex two-dimensional movement sequences along predefined patterns, the resistive method's general principles can also be applied to record simpler movements, e.g. when the rotation around a given axis should be monitored (as during joint flexion/extension or during evaluation of haptic matching performance where two objects need to be aligned within a specified plane).

Beyond the currently intended application, the introduced resistive method furthermore has the potential to be adapted to support general unrestricted pen tracing in two dimensions by measuring the voltages at the four corners of a square resistive layer. In case of more modest requirements on resolution and sampling rate, and depending on space available, a CCD-based readout of traces performed with a light-carrying pen might alternatively be considered.

In summary, the presented setup helps to thoroughly investigate the relation between behavioral and neural manifestations of learning a complex novel movement sequence throughout the entire course of learning and in the context of several paradigmatic variations (e.g. intermanual transfer, passive learning, etc.). By allowing the detailed and temporally high resolved recording of tracing movements during fMRI data acquisition, the introduced method extends the range of phenomena which can be studied in the domains of motor control and learning.

\section{Acknowledgments}

The authors are grateful to J. Grooten, G. Hilgers, R. Lampe and W. Reuter of the RWTH Aachen University for their help during the design and manufacture of the mazes and the maze holder. We also thank P. Gaalman and E. van den Bergh for their assistance in the validation of the presented setup in the MR environment. Furthermore, we acknowledge assistance from $\mathrm{H}$. Hamers and the faculty of Psychology's technical staff at Maastricht University.

\section{References}

Debaere F, Wenderoth N, Sunaert S, Van Hecke P, Swinnen SP. Cerebellar and premotor function in bimanual coordination: parametric neural responses to spatiotemporal complexity and cycling frequency. Neuroimage 2004;21:1416-27.

Illes J, Kirschen MP, Gabrieli JDE. From neuroimaging to neuroethics. Nat Neurosci 2003;6:205.

Jantzen KJ, Steinberg FL, Kelso JAS. Brain networks underlying human timing behavior are influenced by prior context. Proc Natl Acad Sci USA 2004;101:6815-20.

Leube DT, Knoblich G, Erb M, Grodd W, Bartels M, Kircher TTJ. The neural correlates of perceiving one's own movements. Neuroimage 2003;20:2084-90.

Liu JZ, Dai TH, Elster TH, Sahgal V, Brown RW, Yue GH. Simultaneous measurement of human joint force, surface electromyograms, and functional MRI-measured brain activation. J Neurosci Methods 2000;101:49-57.

Mattay VS, Weinberger DR. Organization of the human motor system as studied by functional magnetic resonance imaging. Eur $\mathrm{J}$ Radiol 1999;30:105-14.

Menon RS. Imaging function in the working brain with fMRI. Curr Opin Neurobiol 2001;11:630-6.

Miall RC, Reckess GZ, Imamizu H. The cerebellum coordinates eye and hand tracking movements. Nat Neurosci 2001;4:638-44.

Rowe JB, Frackowiak RSJ. The impact of brain imaging technology on our understanding of motor function and dysfunction. Curr Opin Neurobiol 1999;9:728-34

Sanes JN. Neocortical mechanisms in motor learning. Curr Opin Neurobiol 2003; 13:225-31.

Savoy RL. History and future directions of human brain mapping and functional neuroimaging. Acta Psychol 2001;107:9-42.

van Mier H, Hulstijn W, Petersen SE. Changes in motor planning during the acquisition of movement patterns in a continuous task. Acta Psychol 1993;82:291-312.

van Mier H, Tempel LW, Perlmutter JS, Raichle ME, Petersen SE. Changes in brain activity during motor learning measured with PET: effects of hand of performance and practice. J Neurophysiol 1998;80:2177-99.

van Mier H, Petersen SE. Role of the cerebellum in motor cognition. Ann N Y Acad Sci 2002;978:334-53.

van Mier H, Perlmutter JS, Petersen SE. Functional changes in brain activity during acquisition and practice of movement sequences. Motor Control 2004;8:500-20

Weisskoff RM. Simple measurement of scanner stability for functional NMR imaging of activation in the brain. Magn Reson Med 1996;36:643-5. 